\title{
A Central Potential with a Massive Scalar Field in a Lorentz Symmetry Violation Environment
}

\author{
R. L. L. Vitória (D) and H. Belich \\ Departamento de Física e Química, Universidade Federal do Espírito Santo, Av. Fernando Ferrari, 514, Goiabeiras, 29060-900, \\ Vitória, ES, Brazil \\ Correspondence should be addressed to R. L. L. Vitória; ricardo-luis91@hotmail.com
}

Received 16 January 2019; Accepted 2 May 2019; Published 4 June 2019

Academic Editor: Edward Sarkisyan-Grinbaum

Copyright (C) 2019 R. L. L. Vitória and H. Belich. This is an open access article distributed under the Creative Commons Attribution License, which permits unrestricted use, distribution, and reproduction in any medium, provided the original work is properly cited. The publication of this article was funded by SCOAP ${ }^{3}$.

\begin{abstract}
We investigate the behaviour of a massive scalar field under the influence of a Coulomb-type and central linear central potentials inserted in the Klein-Gordon equation by modifying the mass term in the spacetime with Lorentz symmetry violation. We consider the presence of a background constant vector field which characterizes the breaking of the Lorentz symmetry and show that analytical solutions to the Klein-Gordon equation can be achieved.
\end{abstract}

\section{Introduction}

The Standard Model (SM) presents in a unified way the electromagnetic, weak, and strong nuclear interactions with the exception of gravitational interaction which makes it an incomplete quantum field theory (QFT). In addition, there are some observations, from both a theoretical and observational point of view, about their predictions. Recent experimental data provided measurements of the proton radius which it is different from the value predicted by the SM [1]. There is evidence, by observational data, that the fine structure constant, admitted constant by quantum electrodynamics, one of the pillars of the SM, is changing $[2,3]$. The SM is also limited in explaining the dark sector of the Universe.

Due to these questions about limitations of the SM, it has arisen in recent years interest in investigating the possibility of a physics beyond the SM. In this context, the Lorentz symmetry violation (LSV) has been extensively explored in QFT since their possible scenarios have provided directions in the search for answers about physical effects of possible underlying physical theories, in which they can not be explained or observed through usual physics. In this sense, based on string theory, Kosteleký and Samuel dealt with the spontaneous breaking of symmetry through nonscalar fields, in which the vacuum expected value is constant and acquires a tensorial nature which violates the Lorentz symmetry spontaneously [4]. This possibility of extending the SM was known as the Standard Model Extension (SME) $[5,6]$. In recent decades, the LSV has been extensively studied in various branches of physics [7-61]. Also, the LSV has been studied in nonrelativistic quantum mechanics, for example, Landau-type quantization [62], holonomies [53], and geometric phase [63] and in relativistic quantum mechanics, in particular, on a scalar field [64-67].

In this paper, we have investigated the relativistic quantum dynamics of a scalar field subject to a hard-wall potential and to the Coulomb-type and linear central potentials in a spacetime with LSV. Such configuration in the spacetime, which characterizes the LSV, is provided by the direct coupling between the derivative of the field with an arbitrary constant vector field in the Klein-Gordon equation, where we analyze its effects on a scalar field. Thus, we show that it is possible to find out analytically solutions of bound states and to determine the relativistic energy levels for the scalar field in a Lorentz violating background for each case.

The structure of this paper is as follows: in Section 2, we analyzed the effects of a hard-wall potential on a scalar field in spacetime with LSV caused by the presence of a background constant vector field; in Section 3, we investigated the effect of a Coulomb-type central potential inserted in the Klein-Gordon equation by modifying the mass term and 
discuss their effects on a scalar field in a spacetime with LSV; in Section 4, we insert a linear central potential in the KleinGordon equation by modifying the mass term and determine solutions of bound states for a scalar field in a spacetime with LSV; in Section 5, by modifying the mass term of the KleinGordon equation, we analyzed the effects of a linear plus a Coulomb-type central potential on a scalar field subject to the LSV; in Section 6, we present our conclusions.

\section{Effects of the Hard-Wall Potential}

Effective theories with LSV have been the focus of increasing interest in various physics contexts nowadays. The symmetry that permeates all high energy physics and Lorentz covariance is the basis of the SM of particle physics construction and so it is natural to ask why the interest of this type of violation. Inspired by $[68,69]$, we can write the equation for a scalar field of the form

$$
\left[\square-g\left(v^{\mu} \partial_{\mu}\right)^{2}-m^{2}\right] \phi=0,
$$

where $\square=-\partial^{2} / \partial t^{2}+\nabla^{2}$ is the d'alembertian, $g$ is a coupling constant, $m$ is the rest mass of the scalar field, and $v^{\mu}$ is the background vector field responsible by the LSV. It is important to note that the coupling that appears in (1) conserves the CPT symmetry; that is, it is a CPT-even coupling [68]. In addition, the LSV nonminimal coupling in (1) is analogous to the general structure of (6) of [6], where the background vector field $v^{\mu}$ is associated with a secondorder tensor field $\left(k_{\varphi \varphi}\right)$ from [6]: $v^{a} v^{b} \sim-\left(k_{\varphi \varphi}\right)^{a b}$. Here, we consider the background field vector with the configurations $v^{\mu}=\left(v^{0}, 0\right)$ and $v^{\mu}=(0, \vec{v})$. Note that these particular choices do not prevent us from investigating their effects on the scalar field, since they are possible scenarios of LSV from the theoretical point of view.

In this paper, we work in the Minkowski spacetime with cylindrical symmetry $(c=\hbar=1)$ :

$$
d s^{2}=-d t^{2}+d \rho^{2}+\rho^{2} d \varphi^{2}+d z^{2},
$$

with $\rho=\sqrt{x^{2}+y^{2}}$ being the axial distance.

\subsection{Background Vector Field with the Configuration $v^{\mu}=$} $\left(v^{0}, 0\right)$. From now on, let us make a discussion from the theoretical point of view, where a scalar field is subject to the effects of the LSV given by the presence of the background vector field $v^{\mu}$. In particular, let us consider a background vector field with the following configuration: $v^{\mu}=(a, 0,0,0)$, where $a=$ const. . Note that this is a particular scenario of the LSV. In particular case, (1) becomes

$$
\begin{aligned}
& -\frac{\partial^{2} \phi}{\partial t^{2}}+\frac{\partial^{2} \phi}{\partial \rho^{2}}+\frac{1}{\rho} \frac{\partial \phi}{\partial \rho}+\frac{1}{\rho^{2}} \frac{\partial \phi}{\partial \varphi}+\frac{\partial^{2} \phi}{\partial z^{2}}-g a^{2} \frac{\partial^{2} \phi}{\partial t^{2}}-m^{2} \phi \\
& =0 .
\end{aligned}
$$

Let us consider a particular solution to (3) given in terms of the eigenvalues of the $z$-component of the angular momentum operator $\widehat{L}_{z}=-i \partial_{\varphi}$ and of the eigenvalues of the $z$-component of the linear momentum operator $\widehat{p}_{z}=-i \partial_{z}$ as

$$
\phi(\rho, \varphi, z, t)=e^{-i \mathscr{E} t} e^{i l \varphi} e^{i k z} R(\rho),
$$

where $l=0, \pm 1, \pm 2, \pm 3, \ldots,-\infty<k<\infty$, and $R(\rho)$ is a function of the axial distance. Then, by substituting (4) into (3), we obtain the ordinary differential equation:

$$
\frac{d^{2} R}{d \rho^{2}}+\frac{1}{\rho} \frac{d R}{d \rho}-\frac{l^{2}}{\rho^{2}} R+\alpha^{2} R=0,
$$

where

$$
\alpha^{2}=\left(1+a^{2} g\right) \mathscr{E}^{2}-m^{2}-k^{2} .
$$

Equation (6) is the well-known Bessel differential equation [70]. The general solution to the (6) is given in the form: $R(\rho)=C_{1} J_{|l|}(\alpha \rho)+C_{2} N_{|l|}(\alpha \rho)$, where $J_{|l|}(\alpha \rho)$ and $N_{|l|}(\alpha \rho)$ are the Bessel function of first kind and second kind [70], respectively. The Bessel function of second kind diverges at the origin; then, we must take $C_{2}=0$ in the general solution, since we are interested in a well-behaved solution. Thus, the regular solution to (6) at the origin is given by

$$
R(\rho)=C_{1} J_{|l|}(\alpha \rho) .
$$

Let us restrict the motion of the scalar field to a region where a hard-wall potential is present. This kind of confinement is described by the following boundary condition:

$$
R\left(\rho_{0}\right)=0,
$$

which means that the wave function $R(\rho)$ vanishes at a fixed radius $\rho_{0}$; that is, this boundary condition corresponds to the scalar field subject to a hard-wall potential. The hardwall potential has been studied in Landau-Aharonov-Casher system [71], in a Dirac neutral particle in analogous way to a quantum dot [72], in the relativistic quantum motion of spin0 particles under the influence of noninertial effects in the cosmic string spacetime [73], in the quantum dynamics of scalar bosons [74], in the Aharonov-Bohm effect for bound states in relativistic scalar particle systems in a spacetime with a spacelike dislocation [75] and in rotating effects on the scalar field in the spacetime with linear topological defects [76]. Then, let us consider a particular case where $\alpha \rho_{0} \gg 1$. In this particular case, we can write (7) in the form [70]:

$$
J_{|l|}\left(\alpha \rho_{0}\right) \propto \cos \left(\alpha \rho_{0}-\frac{|l| \pi}{2}-\frac{\pi}{4}\right) .
$$

Hence, by substituting (9) into (7), we obtain from the boundary condition (8) the relativistic energy levels of the system

$$
\begin{aligned}
& \mathscr{E}_{k, l, n} \\
& \approx \pm \sqrt{\frac{1}{\left(1+a^{2} g\right)}\left[m^{2}+k^{2}+\frac{\pi^{2}}{\rho_{0}^{2}}\left(n+\frac{|l|}{2}+\frac{3}{4}\right)^{2}\right]},
\end{aligned}
$$

where $n=0,1,2,3, \ldots$. 
We note that the background that characterizes the LSV caused by the presence of the particular vector field influences the dynamics of the scalar field subject to the hard-wall potential through the presence of the parameters associated with the LSV, $g$ and $a$, on relativistic energy levels of the system. We can also note that, by taking $g \longrightarrow 0$ or $a \longrightarrow$ 0 , we obtain the relativistic energy levels in the Minkowski spacetime.

\subsection{Background Vector Field with the$$
\text { Configuration } v^{\mu}=(0, \vec{v})
$$

2.2.1. The Axial Direction. Let us consider a vector field which governs the LSV with the following configuration: $v^{\mu}=$ $(0, b, 0,0)$, where $b=$ const.. It is important to note that this particular configuration of the vector field that governs the LSV does not arise from the spontaneous breaking of the Lorentz symmetry, since its direction varies. Because it does not have this feature but still is a type of configuration that breaks the violation of the Lorentz symmetry explicitly, it is treated as an external vector field and not as a background vector field. In this particular case, (1) becomes

$$
\begin{aligned}
& -\frac{\partial^{2} \phi}{\partial t^{2}}+\frac{\partial^{2} \phi}{\partial \rho^{2}}+\frac{1}{\rho} \frac{\partial \phi}{\partial \rho}+\frac{1}{\rho^{2}} \frac{\partial \phi}{\partial \varphi}+\frac{\partial^{2} \phi}{\partial z^{2}}-g b^{2} \frac{\partial^{2} \phi}{\partial \rho^{2}} \\
& -m^{2} \phi=0 .
\end{aligned}
$$

Then, by substituting (4) into (11), we have

$$
\left(1-b^{2} g\right) \frac{d^{2} R}{d \rho^{2}}+\frac{1}{\rho} \frac{d R}{d \rho}-\frac{l^{2}}{\rho^{2}} R+\beta^{2} R=0,
$$

where we define

$$
\beta^{2}=\mathscr{E}^{2}-m^{2}-k^{2}
$$

With the purpose of solving (12), let us write

$$
R(\rho)=\rho^{-(1 / 2)\left(b^{2} g /\left(1-b^{2} g\right)\right)} f(\rho) .
$$

Then, by substituting (14) into (12), we obtain the following equation for $f(\rho)$ :

$$
\frac{d^{2} f}{d \rho^{2}}+\frac{1}{\rho} \frac{d f}{d \rho}-\frac{\lambda^{2}}{\rho^{2}} f+\varepsilon^{2} f=0,
$$

where

$$
\begin{aligned}
& \lambda^{2}=\frac{1}{4\left(1-b^{2} g\right)^{2}}\left(4 l^{2}-4 l^{2} b^{2} g+b^{4} g^{2}\right) \\
& \varepsilon^{2}=\frac{\beta^{2}}{\left(1-b^{2} g\right)} .
\end{aligned}
$$

Note that (15) is analogous to (5); then, by following the same steps from (7) to (10), we have

$$
\mathscr{E}_{k, l, n} \approx \pm \sqrt{m^{2}+k^{2}+\frac{\left(1-b^{2} g\right) \pi^{2}}{\rho_{0}^{2}}\left[n+\frac{1}{4\left(1-b^{2} g\right)} \sqrt{4 l^{2}-4 l^{2} b^{2} g+b^{4} g^{2}}+\frac{3}{4}\right]^{2}}
$$

Equation (17) gives us the energy spectrum of a scalar field subject to a hard-wall potential in a spacetime with LSV in the axial direction. We can note that the effects of the LSV influence the quantum dynamics of the scalar field through the presence of parameters $b$ and $g$. In addition, by making $g \longrightarrow 0$ or $b \longrightarrow 0$, we obtain the energy spectrum of a scalar field subject to a hard-wall potential in the Minkowski spacetime.

2.2.2. $z$-Direction. From now on, let us consider the background vector field $v^{\mu}=(0,0,0, c)$, where $c$ is a constant. In this particular case, (1) becomes

$$
\begin{gathered}
-\frac{\partial^{2} \phi}{\partial t^{2}}+\frac{\partial^{2} \phi}{\partial \rho^{2}}+\frac{1}{\rho} \frac{\partial \phi}{\partial \rho}+\frac{1}{\rho^{2}} \frac{\partial \phi}{\partial \varphi}+\frac{\partial^{2} \phi}{\partial z^{2}}-g c^{2} \frac{\partial^{2} \phi}{\partial z^{2}} \\
-m^{2} \phi=0 .
\end{gathered}
$$

We can follow the steps from (3) to (5), where we obtain the differential equation:

$$
\frac{d^{2} R}{d \rho^{2}}+\frac{1}{\rho} \frac{d R}{d \rho}-\frac{l^{2}}{\rho^{2}} R+\epsilon^{2} R=0
$$

with

$$
\epsilon^{2}=\mathscr{E}^{2}-m^{2}-\left(1-c^{2} g\right) k^{2}
$$

We can note that (19) is analogous to (5). Then, by following the same steps from (7) to (10), we obtain

$$
\mathscr{E}_{k, l, n} \approx \pm \sqrt{m^{2}+\left(1-c^{2} g\right) k^{2}+\frac{\pi^{2}}{\rho_{0}^{2}}\left(n+\frac{|l|}{2}+\frac{3}{4}\right)^{2}}
$$

Equation (17) represents the relativistic energy levels of a scalar field subject to a hard-wall potential in the spacetime with LSV governed by a background vector field in the $z$-direction. We can observe that the effects of the LSV influence the quantum dynamics of the scalar field through the presence of an effective linear momentum eigenvalue $k_{\text {eff }}=\sqrt{\left(1-c^{2} g\right)} k$. In addition, by making $g \longrightarrow$ or $c \longrightarrow 0$, we obtain the relativistic energy levels of a scalar field subject to a hard-wall potential in the Minkowski spacetime. 


\section{Effects of the Coulomb-Type Central Potential}

The standard procedure of inserting central potentials into relativistic wave equations, such as the Klein-Gordon and Dirac equations, is through the minimum coupling which is represented by the transformation in the linear momentum operator, $\widehat{p}_{\mu} \longrightarrow \widehat{p}_{\mu}-q A_{\mu}$, by $q A_{0}=V(\vec{r})=V(r)$. A well-known example, in a system of spherical symmetry, is the Coulomb potential in the description of the hydrogen atom and in the piônic atom [77]. Another procedure to insert central potentials in relativistic wave equations, in particular in the Klein-Gordon equation, is by modifying the mass term of the equation, as shown in [77]. Recently, through the modification of the mass term of the Klein-Gordon equation, some studies have been done in the context of quantum mechanics, for example, in exact solutions of the massdependent Klein-Gordon equation with the vector quarkantiquark interaction and harmonic oscillator potential [78], in the relativistic quantum dynamics of a charged particle in cosmic string spacetime in the presence of magnetic field and scalar potential [79], in the linear confinement of a scalar particle in a Gödel-type spacetime [80], and in exact solutions of the Klein-Gordon equation in the presence of a dyon, magnetic flux, and scalar potential in the spacetime of gravitational defects [81]. In this section, we take into account a scalar potential proportional to the inverse of the axial distance. It is important to mention that the Coulombtype potential has been studied under the effects of the Klein-Gordon oscillator [82, 83], in a Dirac particle [84], with propagation of gravitational waves [85], in condensed matter systems, such as 1-dimensional systems [86-88], pseudoharmonic interactions [89, 90], and molecules [91-93]. Then, inspired by [77], we introduce a Coulomb-type central potential into the Klein-Gordon equation by modifying the mass term, $m \longrightarrow m+U(\vec{r})$, where $m$ is a constant that corresponds to the rest mass of the scalar field and $U(\vec{r})$ is a scalar potential, with the intention of confining the scalar field in a spacetime with LSV and investigating the effects of the Coulomb-type central potential and spacetime anisotropies generated by the background vector field $v^{\mu}$ on the relativisitic quantum dynamics of a scalar field. Then, the mass term of the Klein-Gordon equation becomes $m \longrightarrow m+v / \rho$, where $v$ is a constant that characterizes the Coulomb-type central potential. In this way, (1) takes the form

$$
\left[\square-g\left(v^{\mu} \partial_{\mu}\right)^{2}-\left(m+\frac{v}{\rho}\right)^{2}\right] \phi=0 .
$$

3.1. Background Vector Field with the Configuration $v^{\mu}=$ $\left(v^{0}, 0\right)$. Let us consider a background vector field with the following configuration: $v^{\mu}=(a, 0,0,0)$. In this particular case, (22) becomes

$$
-\frac{\partial^{2} \phi}{\partial t^{2}}+\frac{\partial^{2} \phi}{\partial \rho^{2}}+\frac{1}{\rho} \frac{\partial \phi}{\partial \rho}+\frac{1}{\rho^{2}} \frac{\partial \phi}{\partial \varphi}+\frac{\partial^{2} \phi}{\partial z^{2}}-g a^{2} \frac{\partial^{2} \phi}{\partial t^{2}}
$$

$$
-\frac{2 m \nu}{\rho} \phi-\frac{\nu^{2}}{\rho^{2}} \phi-m^{2} \phi=0 .
$$

By following the steps from (3) to (5), we obtain the axial wave equation:

$$
\frac{d^{2} R}{d \rho^{2}}+\frac{1}{\rho} \frac{d R}{d \rho}-\frac{\gamma^{2}}{\rho^{2}} R-\frac{2 m \nu}{\rho} R-\bar{\alpha}^{2} R=0,
$$

where

$$
\begin{aligned}
& \bar{\alpha}^{2}=m^{2}+k^{2}-\left(1+a^{2} g\right) \mathscr{E}^{2} ; \\
& \gamma^{2}=l^{2}+v^{2} .
\end{aligned}
$$

Let us define $r=2 \bar{\alpha} \rho$; then, (25) becomes

$$
\frac{d^{2} R}{d r^{2}}+\frac{1}{r} \frac{d R}{d r}-\frac{\gamma^{2}}{r^{2}} R+\frac{\delta}{r} R-\frac{1}{4} R=0,
$$

where

$$
\delta=\frac{m|\nu|}{\alpha}
$$

Next, let us impose that $R(r) \longrightarrow 0$ when $r \longrightarrow 0$ and $r \longrightarrow$ $\infty$. In this way, the radial wave function can be written as

$$
R(r)=r^{|\gamma|} e^{-(1 / 2) r} F(r),
$$

And then, we obtain the following equation for $F(r)$ :

$$
r \frac{d^{2} F}{d r^{2}}+(2|\gamma|+1-r) \frac{d F}{d r}+\left(\delta-\frac{1}{2}-|\gamma|\right) F=0
$$

which is called in literature the confluent hypergeometric equation and $F(r)={ }_{1} F_{1}(|\gamma|+1 / 2-\delta, 2|\gamma|+1 ; r)$ is the confluent hypergeometric function [70]. It is well known that the confluent hypergeometric series becomes a polynomial of degree $n$ by imposing that $|\gamma|+1 / 2-\delta=-n$, where $n=0,1,2,3, \ldots$. With this condition, we obtain

$$
\begin{aligned}
& \mathscr{E}_{k, l, n} \\
& = \pm \sqrt{\frac{1}{\left(1+a^{2} g\right)}\left[m^{2}+k^{2}-\frac{m^{2} v^{2}}{\left(n+\sqrt{l^{2}+v^{2}}+1 / 2\right)^{2}}\right]} .
\end{aligned}
$$

Hence, by introducing the scalar potential by modification of the mass term, we can note, through (30) which represents the relativistic energy levels of the scalar field, that the spectrum of energy is modified by the influence of the Coulomb-type central potential. Note also that the spacetime with LSV influences energy levels through the presence of parameters $a$ and $g$. By making $a \longrightarrow 0$ or $g \longrightarrow 0$, we have the relativistic energy levels of the scalar field subject to the Coulomb-type central potential in the Minkowski spacetime. 


\subsection{Background Vector Field with the Configuration $v^{\mu}=(0, \vec{v})$}

3.2.1. The Axial Direction. Let us consider an external vector field with the following configuration: $v^{\mu}=(0, b, 0,0)$. In this particular case, the axial wave equation becomes

$$
\left(1-b^{2} g\right) \frac{d^{2} R}{d \rho^{2}}+\frac{1}{\rho} \frac{d R}{d \rho}-\frac{\gamma^{2}}{\rho^{2}} R-\frac{2 m \nu}{\rho} R+\beta^{2} R=0,
$$

where $\beta^{2}$ and $\gamma^{2}$ are defined in (13) and (25), respectively. By substituting (14) into (31), we obtain

$$
\frac{d^{2} f}{d \rho^{2}}+\frac{1}{\rho} \frac{d f}{d \rho}-\frac{\iota^{2}}{\rho^{2}} f-\frac{2 m \nu}{\left(1-b^{2} g\right) \rho} f-\bar{\varepsilon}^{2} f=0,
$$

where

$$
\begin{aligned}
\iota^{2} & =\frac{1}{4\left(1-b^{2} g\right)^{2}}\left(4 \gamma^{2}-4 \gamma^{2} b^{2} g+b^{4} g^{2}\right) ; \\
\bar{\varepsilon} & =\frac{\beta^{2}}{b^{2} g-1} .
\end{aligned}
$$

Equation (32) is analogous to (26). Then, by following the same steps from (28) to (30), we have

$$
\mathscr{E}_{k, l, n}= \pm \sqrt{m^{2}+k^{2}-\left(1-b^{2} g\right) \frac{m^{2} v^{2}}{(n+|\iota|+1 / 2)^{2}}}
$$

which is the energy spectrum of a scalar field subject to the Coulomb-type central potential in a spacetime with LSV generated by an external external vector field in the axial direction. Note that the nature of the external vector field influences energy levels through the presence of the parameters associated with the LSV, $b$ and $g$. By making $b \longrightarrow$ 0 or $g \longrightarrow 0$, we obtain the relativistic energy levels of a scalar field subject to the Coulomb-type central potential in the Minkowski spacetime.

3.2.2. $z$-Direction. Let us consider a background vector field with the following configuration: $v^{\mu}=(0,0,0, c)$. In this particular case, the axial wave equation becomes

$$
\frac{d^{2} R}{d \rho^{2}}+\frac{1}{\rho} \frac{d R}{d \rho}-\frac{\gamma^{2}}{\rho^{2}} R-\frac{2 m \nu}{\rho} R-\bar{\epsilon}^{2} R=0
$$

where $\gamma^{2}$ is defined in (25) and

$$
\bar{\epsilon}^{2}=m^{2}+\left(1-c^{2} g\right) k^{2}-\mathscr{E}^{2}
$$

We can note that (35) is analogous to (24). Then, by following the same steps from (24) to (30), we obtain

$$
\begin{aligned}
& \mathscr{E}_{k, l, n} \\
& = \pm \sqrt{m^{2}+\left(1-c^{2} g\right) k^{2}-\frac{m^{2} v^{2}}{\left(n+\sqrt{l^{2}+v^{2}}+1 / 2\right)^{2}}}
\end{aligned}
$$

which is the general expression for the relativistic energy levels for the scalar field with position-dependent mass described in (22) in the spacetime with LSV. We can observe the influence of the LSV in (37) through the presence of the parameters $c$ and $g$. They yield a shift in the linear momentum eigenvalue that gives rise to an effective linear momentum quantum number $k_{\text {eff }}=\sqrt{1-c^{2} g} k$. In addition, by making $c \longrightarrow 0$ and $g \longrightarrow 0$, we obtain the relativistic energy levels of a scalar field subject to the Coulomb-type central potential in the Minkowski spacetime.

\section{Effects of the Linear Central Potential}

In this section, we analyse the relativistic quantum effects of a linear central potential, through the modification of the mass term [77] as $m \longrightarrow m+\mu \rho[66,79]$, where $\mu$ is a constant, and the effects of the LSV on the scalar field. The linear central potential has been studied and investigated under the effects of the Klein-Gordon oscillator [94], in the relativistic quantum dynamics of a scalar particle in the spacetime with torsion [95], and in Majorana fermion [96]. In this way, the a Klein-Gordon equation (1) becomes

$$
\left[\square-g\left(v^{\mu} \partial_{\mu}\right)^{2}-(m+\mu \rho)^{2}\right] \phi=0 .
$$

4.1. Background Vector Field with the Configuration $v^{\mu}=$ $\left(v^{0}, 0\right)$. Let us consider a background vector field with the following configuration: $v^{\mu}=(a, 0,0,0)$. In this particular case, the axial wave equation becomes

$$
\frac{d^{2} R}{d \rho^{2}}+\frac{1}{\rho} \frac{d R}{d \rho}-\frac{l^{2}}{\rho^{2}} R-2 m \mu \rho R-\mu^{2} \rho^{2} R+\alpha^{2} R=0,
$$

where $\alpha^{2}$ is defined in (6).

From now on, let us consider $\xi=\sqrt{\mu} \rho$; thus, we rewrite (39) in the form

$$
\frac{d^{2} R}{d \xi^{2}}+\frac{1}{\xi} \frac{d R}{d \xi}-\frac{l^{2}}{\xi^{2}} R-\eta \xi R-\xi^{2} R+\frac{\alpha^{2}}{\eta} R=0,
$$

where we define the new parameter

$$
\eta=\frac{2 m}{\sqrt{\mu}}
$$

By analysing the asymptotic behaviour at $\xi \longrightarrow 0$ and $\xi \longrightarrow$ $\infty$, then, we can write the function $R(\xi)$ in terms of an unknown function $H(\xi)$ in the form $[66,67,79]$ :

$$
R(\xi)=\xi^{l} e^{-(1 / 2) \xi(\xi+\eta)} H(\xi),
$$

and thus, by substituting (42) into (41), we can observe that the function $H(\xi)$ is a solution to the following second-order differential equation:

$$
\frac{d^{2} H}{d \xi^{2}}+\left[\frac{(2|l|+1)}{\xi}-\eta-2 \xi\right] \frac{d H}{d \xi}+\left[h-\frac{g}{\xi}\right] H=0,
$$


where

$$
\begin{aligned}
& h=\frac{\alpha^{2}}{\mu}-2-2|l|+\frac{\eta^{2}}{4} ; \\
& g=\frac{\eta}{2}(2|l|+1) .
\end{aligned}
$$

Equation (44) is called in the literature the biconfluent Heun equation $[79,97]$ and the function $H(\xi)=H_{B}\left(2|l|, \eta, \alpha^{2} / \mu+\right.$ $\left.\eta^{2} / 4,0 ; \xi\right)$ is the biconfluent Heun function.

Let us search for polynomial solutions to (43); then, for this purpose, we write the solution to (43) as a power series expansion around the origin, which is a regular singular point [79]:

$$
H(\xi)=\sum_{j=0}^{\infty} d_{j} \xi^{j}
$$

By substituting this series into (43), we obtain the recurrence relation:

$$
d_{j+2}=\frac{[g+\eta(j+1)] d_{j+1}-(h-2 j) d_{j}}{(j+2)(j+2+2|l|)},
$$

where the coefficients $d_{1}$ and $d_{2}$ are

$$
\begin{aligned}
d_{1} & =\frac{g}{(1+2|l|)} d_{0}=\frac{\eta}{2} \\
d_{2} & =\frac{(g+\eta) d_{1}-h d_{0}}{2(2+2|l|)} \\
& =\frac{1}{4(1+|l|)}\left[\frac{\eta^{2}}{4}(2|l|+3)-h\right],
\end{aligned}
$$

with $d_{0}=1$.

In search of polynomial solutions to the biconfluent Heun equation (43), we can note from (45) that the biconfluent Heun series becomes a polynomial of degree $\bar{n}$ when [79]

$$
\begin{gathered}
h=2 \bar{n} ; \\
d_{\bar{n}+1},
\end{gathered}
$$

where $\bar{n}=1,2,3,4, \ldots$. Therefore, the condition $h=2 \bar{n}$ gives the expression

$$
\mathscr{E}_{k, l, \bar{n}}= \pm \sqrt{\frac{1}{\left(1+a^{2} g\right)}\left[k^{2}+2 \mu(1+\bar{n}+|l|)\right]} .
$$

However, our analysis is not complete, since condition $d_{\bar{n}+1}=0$ must be analyzed and this can only be attributed by values of $\bar{n}$ in it. In this case, considering $\bar{n}=1$, which from the physical point of view represents the lowest energy state of the system and choosing the parameter associated with the linear potential $\mu=\mu_{k, l, \bar{n}}$ to adjust condition $d_{\bar{n}+1}=0$, that is, $d_{\bar{n}+1}=d_{2}=0$, not only for $\bar{n}=1$ but also for any value of $\bar{n}$, we obtain the allowed values from $\mu$ to the radial mode $\bar{n}=1$ :

$$
\mu_{l, 1}=\frac{m^{2}}{2}(2|l|+3) \text {. }
$$

With the relation given in (50), we have that the possible values of the parameter $\mu$ are determined by the quantum numbers $\{l, \bar{n}\}$ of the system. By substituting (50) into (49), the allowed energies for the lowest energy state are given by

$$
\mathscr{E}_{k, l, 1}= \pm \sqrt{\frac{1}{\left(1+a^{2} g\right)}\left[m^{2}(3+2|l|)(2+|l|)+k^{2}\right]} .
$$

It is important to note that, unlike the previous cases analyzed, it is not possible to determine a closed solution for the biconfluent Heun polynomials for its more general case of its asymptotic behavior, that is, for large values of its argument. Hence, through the two conditions given in the (48), arising from truncation of the power series (45), it is only possible to determine polynomial solutions separately for each radial mode $\bar{n}$ of the system, as discussed in the [79, 97-99]. From the physical point of view, this quantum effect arises due to the presence of the linear central potential in the system. Besides, we can note the influence of the LSV in (51) through the presence of the parameters $a$ and $g$. By taking $a \longrightarrow 0$ or $g \longrightarrow 0$ in (51), we obtain the allowed energies for the lowest energy state for the position-dependent mass system in the Minkowski spacetime.

\subsection{Background Vector Field with the \\ Configuration $v^{\mu}=(0, \vec{v})$}

4.2.1. The Axial Direction. Let us consider an external vector field with the following configuration: $v^{\mu}=(0, b, 0,0)$. In this particular case, the axial wave equation becomes

$$
\begin{gathered}
\left(1-b^{2} g\right) \frac{d^{2} R}{d \rho^{2}}+\frac{1}{\rho} \frac{d R}{d \rho}-\frac{l^{2}}{\rho^{2}} R-2 m \mu \rho R-\mu^{2} \rho^{2} R \\
+\beta^{2} R=0,
\end{gathered}
$$

where $\beta^{2}$ is defined in (13). By substituting (14) into (52), we obtain

$$
\begin{aligned}
\frac{d^{2} f}{d \rho^{2}} & +\frac{1}{\rho} \frac{d f}{d \rho}-\frac{\lambda^{2}}{\rho^{2}} f-\frac{2 m \mu \rho}{\left(1-b^{2} g\right)} f-\frac{\mu^{2} \rho^{2}}{\left(1-b^{2} g\right)} f \\
& +\frac{\beta^{2}}{\left(1-b^{2} g\right)} f=0,
\end{aligned}
$$

where $\lambda^{2}$ is defined in (16).

Let us define $s=\left(\sqrt{\mu} /\left(1-b^{2} g\right)^{1 / 4}\right) \rho$; then, (53) becomes

$$
\frac{d^{2} f}{d s^{2}}+\frac{1}{s} \frac{d f}{d s}-\frac{\lambda^{2}}{s^{2}}-\bar{\eta} s f-s^{2} f+\Lambda f=0,
$$

where

$$
\begin{aligned}
& \bar{\eta}=\frac{2 m}{\sqrt{\mu}\left(1-b^{2} g\right)^{1 / 4}} \\
& \Lambda=\frac{\beta^{2}}{\mu \sqrt{1-b^{2} g}} .
\end{aligned}
$$


We can note that (55) is analogous to (40). Then, by following the steps from (42) to (46), we obtain the recurrence relation:

$$
d_{j+2}=\frac{[\bar{g}+\bar{\eta}(j+1)] d_{j+1}-(\bar{h}-2 j) d_{j}}{(j+2)(j+2+2|l|)},
$$

with the relations

$$
\begin{aligned}
& d_{1}=\frac{\bar{g}}{(1+2|\lambda|)}=\frac{\bar{\eta}}{2} \\
& d_{2}=\frac{1}{4(1+|\lambda|)}\left[\frac{\bar{\eta}^{2}}{4}(2|\lambda|+1)+\frac{\bar{\eta}^{2}}{2}-\bar{h}\right],
\end{aligned}
$$

where we are considering $d_{0}=1$ and define the new parameters

$$
\begin{aligned}
& \bar{h}=\Lambda-2-2|\lambda|+\frac{\bar{\eta}^{2}}{4} ; \\
& \bar{g}=\frac{\bar{\eta}}{2}(2|\lambda|+1) .
\end{aligned}
$$

In search of a polynomial solution to the function $f(s)$, we have that the polynomial of degree $\bar{n}$ to $f(s)$ is achieved when we impose that [79]

$$
\bar{h}=2 \bar{n} ;
$$

$$
d_{\bar{n}+1}=0 \text {, }
$$

where $\bar{n}=1,2,3,4, \ldots$ From the condition $\bar{h}=2 \bar{n}$, we have the expression

$$
\mathscr{E}_{k, l, \bar{n}}= \pm \sqrt{k^{2}+2 \sqrt{1-b^{2} g} \mu_{k, l, \bar{n}}(1+\bar{n}+|\lambda|)}
$$

where we have labelled $\mu=\mu_{k, l, \bar{n}}$ as in the previous section. Further, let us analyse the condition $d_{\bar{n}+1}=0$ by working with the lowest energy state $\bar{n}=1$. In this case, we have that $d_{\bar{n}+1}=$ $d_{2}=0$, and then, the possible values of the parameter $\mu$ are determined by

$$
\mu_{l, 1}=\frac{m^{2}(2|\lambda|+3)}{2 \sqrt{1-b^{2} g}} .
$$

Note that the allowed values of $\mu$ are determined by the quantum numbers of the system $\{l, \bar{n}\}$ and parameters associated with the LSV, $b$ and $g$, in contrast with the previous subsection (50). Hence, the allowed energies for the lowest energy state $(\bar{n}=1)$ are

$$
\mathscr{E}_{k, l, 1}= \pm \sqrt{m^{2}\left[3+\frac{\sqrt{4 l^{2}-4 l^{2} b^{2} g+b^{4} g^{2}}}{\left(1-b^{2} g\right)}\right]\left[2+\frac{\sqrt{4 l^{2}-4 l^{2} b^{2} g+b^{4} g^{2}}}{2\left(1-b^{2} g\right)}\right]+k^{2}}
$$

Equation (62) is the expression of the relativistic energy levels of the lowest energy state, $\bar{n}=1$, for a scalar field subject to a linear central potential in the spacetime with LSV caused by the presence of an external vector field. We can observe the influence of the LSV in (61) and (62) through the presence of the parameters $b$ and $g$. In addition, by making $b \longrightarrow 0$ or $g \longrightarrow 0$, we obtain the expression of the relativistic energy level of the lowest energy state in the Minkowski spacetime.

4.2.2. $z$-Direction. Now, let us consider the configuration of the vector field given in the form $v^{\mu}=(0,0,0, c)$. In this particular case, the axial wave equation becomes

$$
\frac{d^{2} R}{d \rho^{2}}+\frac{1}{\rho} \frac{d R}{d \rho}-\frac{l^{2}}{\rho^{2}} R-2 m \mu \rho R-\mu^{2} \rho^{2} R+\epsilon^{2} R=0,
$$

where $\epsilon^{2}$ is defined in (20).

Equation (63) is analogous to (39). Then, following the same steps from (39) to (51), we obtain

$$
\mathscr{E}_{k, l, 1}= \pm \sqrt{m^{2}(3+2|l|)(2+|l|)+\left(1-c^{2} g\right) k^{2}}
$$

which is the general expression for the allowed energies for the lowest energy state for the position-dependent mass system described in (38) in the spacetime with LSV. We can note the influence of the spacetime with LSV in (64) through the presence of the parameters $c$ and $g$. They yield a shift in the linear momentum quantum number that gives rise to an effective linear momentum quantum number $k_{\text {eff }}=$

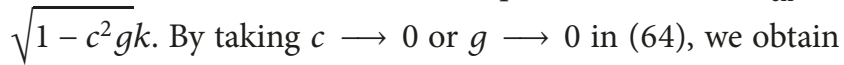
the relativistic allowed energy levels for $\bar{n}=1$ of a scalar field subject to the linear central potential in the Minkowski spacetime.

\section{Effects of the Coulomb-Type Plus Linear Central Potential}

In this section, let us consider the scalar field in $(3+1)$ dimensions given in (1) under the influence of the linear and Coulomb-type potentials, where they are inserted into the Klein-Gordon equation by modifying the mass term, $m \longrightarrow$ $m+\mu \rho+\nu / \rho$, that is, a Cornell-type potential [78], since we are working with cylindrical symmetry. This type of potential 
has been studied in $[79,81,94]$. In this way, the Klein-Gordon equation (1) becomes

$$
\left[\square-\left(v^{\mu} \partial_{\mu}\right)^{2}-\left(m+\mu \rho+\frac{\nu}{\rho}\right)^{2}\right] \phi=0 .
$$

5.1. Background Vector Field with the Configuration $v^{\mu}=$ $\left(v^{0}, 0\right)$. Let us consider the background vector field $v^{\mu}=$ $(a, 0,0,0)$. In this particular case, (65) gives axial wave equation:

$$
\begin{aligned}
\frac{d^{2} R}{d \rho^{2}} & +\frac{1}{\rho} \frac{d R}{d \rho}-\frac{\gamma^{2}}{\rho^{2}} R-\frac{2 m \nu}{\rho} R-2 m \mu \rho R-\mu^{2} \rho^{2} R \\
& +\tilde{\alpha}^{2} R=0,
\end{aligned}
$$

where $\gamma^{2}$ is defined in (25) and we define the new parameter

$$
\widetilde{\alpha}^{2}=\left(1+a^{2} g\right) \mathscr{E}^{2}-m^{2}-k^{2}-2 \mu \nu
$$

From now on, let us consider $\xi=\sqrt{\mu} \rho$; thus, we rewrite (67) in the form

$$
\frac{d^{2} R}{d \xi^{2}}+\frac{1}{\xi} \frac{d R}{d \xi}-\frac{\gamma^{2}}{\xi^{2}} R-\frac{\zeta}{\xi} R-\eta \xi R-\xi^{2} R+\frac{\widetilde{\alpha}^{2}}{\mu} R=0,
$$

where $\eta$ is defined in (41) and

$$
\zeta=\frac{2 m \nu}{\sqrt{\mu}}
$$

By analysing the asymptotic behaviour of the possible solutions to $(68)$ at $\xi \longrightarrow 0$ and $\xi \longrightarrow \infty$, we can write the function $R(\xi)$ in terms of an unknown function $G(\xi)$ as

$$
R(\xi)=\xi^{|\gamma|} e^{-(1 / 2) \xi(\xi+\eta)} G(\xi)
$$

Then, by substituting (70) into (68), we obtain the biconfluent Heun equation $[79,97]$ :

$$
\frac{d^{2} G}{d \xi^{2}}+\left[\frac{(2|\gamma|+1)}{\xi}-\eta-2 \xi\right] \frac{d G}{d \xi}+\left[\tilde{h}-\frac{\tilde{g}}{\xi}\right] G=0,
$$

where

$$
\begin{aligned}
& \tilde{h}=\frac{\tilde{\alpha}^{2}}{\mu}+\frac{\eta^{2}}{4}-2-2|\gamma| ; \\
& \tilde{g}=\frac{\eta}{2}(2|\gamma|+1)+\zeta,
\end{aligned}
$$

and the function $G(\xi)$ is the biconfluent Heun function, $G(\xi)=G_{B}\left(2|\gamma|, \eta, \widetilde{\alpha}^{2} / \mu+\eta^{2} / 4,2 \zeta ; \xi\right)$.
Further, by using the Fröbenius method as in (45) and (46), we obtain the recurrence relation:

$$
d_{j+2}=\frac{[\widetilde{g}+\eta(j+1)] d_{j+1}-(\widetilde{h}-2 j) d_{j}}{(j+2)(j+2+2|\gamma|)},
$$

with the relations

$$
\begin{aligned}
d_{1} & =\frac{\tilde{g}}{1+2|\gamma|} ; \\
d_{2} & =\frac{1}{4(1+|\gamma|)}\left[(\tilde{g}+\eta) d_{1}-\tilde{h}\right] \\
& =\frac{1}{4(1+|\gamma|)}\left[\frac{\tilde{g}^{2}+\eta \tilde{g}}{1+2|\gamma|}-\tilde{h}\right],
\end{aligned}
$$

where we are considering $d_{0}=1$.

As seen in Section 4, through the recurrence relation (73), we can see that the power series expansion (73) becomes a polynomial of degree $\bar{n}$ by imposing two conditions [79]:

$$
\begin{aligned}
\tilde{h} & =2 \bar{n} ; \\
d_{\bar{n}+1} & =0,
\end{aligned}
$$

where $\bar{n}=1,2,3,4, \ldots$ From the condition $\widetilde{h}=2 \bar{n}$, we obtain the expression

$$
\mathscr{E}_{k, l, \bar{n}}= \pm \sqrt{\frac{1}{\left(1+a^{2} g\right)}\left[k^{2}+2 \mu_{k, l, \bar{n}}(1+\bar{n}+|\gamma|+\nu)\right]}
$$

For our analysis to be complete, it is necessary to analyze the condition $d_{\bar{n}+1}=0$, which, as already seen in Section 4, the parameter associated with the linear central potential is chosen to adjust such condition $\left(\mu=\mu_{k, l, \bar{n}}\right)$. Then, for $\bar{n}=1$, we have $d_{\bar{n}+1}=d_{2}=0$, which gives the allowed values of $\mu$ for $\bar{n}=1$ :

$$
\begin{aligned}
\mu_{l, 1}= & \frac{m^{2}}{2}\left(2 \sqrt{l^{2}+v^{2}}+3\right)+4 m^{2} v \frac{\left(\sqrt{l^{2}+v^{2}}+1\right)}{\left(2 \sqrt{l^{2}+v^{2}}+1\right)} \\
& +\frac{2 m^{2} v^{2}}{\left(2 \sqrt{l^{2}+v^{2}}+1\right)} .
\end{aligned}
$$

By comparing the expressions for the allowed values of $\mu$ (50) and (77), we have that the presence of the Coulomb-type potential modifies the the expression for the allowed values of $\mu$ for $\bar{n}=1$; that is, the possible values of $\mu$ are determined by the parameter associated with the Coulomb-type potential, and by the the quantum numbers of the system $\{l, n\}$. With the result given in (77), the allowed energies for the lowest energy state are

$$
\mathscr{E}_{k, l, 1}= \pm \sqrt{\frac{1}{\left(1+a^{2} g\right)}\left\{k^{2}+m^{2}\left[(2|\gamma|+3)+8 \nu \frac{(|\gamma|+1)}{(2|\gamma|+1)}+\frac{4 \nu^{2}}{(2|\gamma|+1)}\right](2+|\gamma|+\nu)\right\}}
$$


We can note that the allowed energy values for $\bar{n}=1$ are influenced by the spacetime with LSV through the presence of the constant parameters $a$ and $g$. Note that, by making $v=$ 0 in (77) and (78), we recover (50) and (51), respectively. In addition, by making $a \longrightarrow 0$ or $g \longrightarrow 0$, we obtain the allowed energy values of a scalar field subject to the Coulomb-type plus linear central potential in the Minkowski spacetime.

\subsection{Background Vector Field with the \\ Configuration $v^{\mu}=(0, \vec{v})$}

5.2.1. The Axial Direction. Let us consider the external vector field $v^{\mu}=(0, b, 0,0)$. In this particular case, $(65)$ gives axial wave equation:

$$
\begin{gathered}
\left(1-b^{2} g\right) \frac{d^{2} R}{d \rho^{2}}+\frac{1}{\rho} \frac{d R}{d \rho}-\frac{\gamma^{2}}{\rho^{2}} R-\frac{2 m \nu}{\rho} R-2 m \mu \rho R \\
-\mu^{2} \rho^{2} R+\bar{\beta}^{2} R=0,
\end{gathered}
$$

where $\gamma^{2}$ is defined in (25) and

$$
\bar{\beta}^{2}=\mathscr{E}^{2}-m^{2}-k^{2}-2 \mu \nu
$$

By substituting (14) into (79), we obtain

$$
\begin{aligned}
\frac{d^{2} f}{d \rho^{2}} & +\frac{1}{\rho} \frac{d f}{d \rho}-\frac{\iota^{2}}{\rho^{2}} f-\frac{2 m \nu}{\left(1-b^{2} g\right) \rho} f-\frac{2 m \mu}{\left(1-b^{2} g\right)} \rho f \\
& -\frac{\mu^{2}}{\left(1-b^{2} g\right)} f+\frac{\bar{\beta}^{2}}{\left(1-b^{2} g\right)} f=0,
\end{aligned}
$$

where $\iota^{2}$ is defined in (33). Now, by using the variable change $s=\left(\sqrt{\mu} /\left(1-b^{2} g\right)^{1 / 4}\right) \rho$, we have

$$
\frac{d^{2} f}{d s^{2}}+\frac{1}{s} \frac{d f}{d s}-\frac{\iota^{2}}{s^{2}} f-\frac{\bar{\zeta}}{s} f-\bar{\eta} s f-s^{2} f+\bar{\Lambda} f=0
$$

where $\bar{\eta}$ is defined in (55) and

$$
\begin{aligned}
& \bar{\zeta}=\frac{2 m \nu}{\sqrt{\mu}\left(1-b^{2} g\right)^{1 / 4}} \\
& \bar{\Lambda}=\frac{\bar{\beta}^{2}}{\mu\left(1-b^{2} g\right)}
\end{aligned}
$$

We can note that (82) is analogous to (68). Then, let us follow the steps from (70) to (76), and we obtain the expression

$$
\mathscr{E}_{k, l, \bar{n}}= \pm \sqrt{k^{2}+2 \sqrt{1-b^{2} g} \mu_{k, l, \bar{n}}(1+\bar{n}+|\iota|+\nu)}
$$

As we have discussed in the previous section, the parameter $\mu$ can be adjusted to satisfy the truncation conditions of the biconfluent Heun series. Then, for $\bar{n}=1$, we obtain the allowed values of $\mu$ :

$$
\begin{aligned}
\mu_{l, 1}= & \frac{m^{2}(2|\iota|+3)}{2 \sqrt{1-b^{2} g}}+\frac{4 m^{2} v(|\iota|+1)}{\left(1-b^{2} g\right)(1+2|\iota|)} \\
& +\frac{2 m^{2} v^{2}}{\left(1-b^{2} g\right)^{3 / 2}(1+2|\iota|)} .
\end{aligned}
$$

We can observe that, in contrast to Section 5.1, the configuration of the external vector field that characterizes the LSV modifies the allowed values of $\mu$ for $n=1$. By making $\nu=0$, we recover (61). Hence, the allowed values of the relativistic energy for the radial mode $\bar{n}=1$ are

$$
\mathscr{E}_{k, l, 1}= \pm \sqrt{k^{2}+m^{2}\left[(2|\iota|+3)+\frac{8 v(1+|\iota|)}{\sqrt{1-b^{2} g}(1+2|\iota|)}+\frac{4 v^{2}}{\left(1-b^{2} g\right)(1+2|\iota|)}\right](2+|\iota|+\nu)} .
$$

We can note the influence of the LSV in (86) through the presence of the parameters $b$ and $g$. In addition, by making $v=0$, we recover (62). By talking $b \longrightarrow 0$ or $g \longrightarrow 0$, we obtain the allowed energy for $\bar{n}=1$ of a scalar field subject to the Coulomb-type plus linear central potential in the Minkowski spacetime.

5.2.2. $z$-Direction. Let us consider the background vector field $v^{\mu}=(0,0,0, c)$. In this particular case, (65) gives axial wave equation becomes

$$
\begin{aligned}
\frac{d^{2} R}{d \rho^{2}} & +\frac{1}{\rho} \frac{d R}{d \rho}-\frac{\gamma^{2}}{\rho^{2}} R-\frac{2 m \nu}{\rho} R-2 m \mu \rho R-\mu^{2} \rho^{2} R \\
& +\widetilde{\epsilon}^{2} R=0,
\end{aligned}
$$

where $\gamma^{2}$ is defined in (25) and

$$
\widetilde{\alpha}^{2}=\mathscr{E}^{2}-m^{2}-\left(1-c^{2} g\right) k^{2}-2 \mu \nu
$$

Equation (87) is analogous to (66). Then, by following the same steps from (66) to (78), we obtain

$$
\mathscr{E}_{k, l, 1}= \pm \sqrt{\left(1-c^{2} g\right) k^{2}+m^{2}\left[(2|\gamma|+3)+\frac{8 \nu(|\gamma|+1)}{(2|\gamma|+1)}+\frac{4 \nu^{2}}{(2|\gamma|+1)}\right](2+|\gamma|+\nu)}
$$


which is the general expression for the allowed energies for the lowest energy state of the system described in (65) in the spacetime with LSV. We can note the influence of the LSV in the (89) through the presence of the parameters $c$ and $g$. They yield a shift in the linear momentum eigenvalue that gives rise to an effective linear momentum quantum number $k_{\text {eff }}=\sqrt{1-c^{2} g} k$. In addition, by making $v=0$ in (89), we recover (64). By taking $c \longrightarrow 0$ or $g \longrightarrow 0$ in (89), we obtain the allowed energy values for $\bar{n}=1$ of a scalar field subject to the Coulomb-type plus linear central potential in the Minkowski spacetime.

\section{Conclusions}

We have investigated the effects of the LSV on a scalar field subject to a hard-wall potential and Coulomb-type and linear central potentials. The LSV is governed by the presence of a background constant vector field which modifies the structure of the Klein-Gordon equation (1) by being directly coupled to a derivative of the field. This coupling conserves the CPT symmetry, so it is considered a CPT-even coupling [68]. For our analysis, we consider the particular cases where the vector field has the particular configurations $v^{\mu}=\left(v^{0}, 0\right)$ and $v^{\mu}=(0, \vec{v})$. In the particular case where the vector field has the particular configuration $v^{\mu}=(0, \vec{v})$, it is possible to note that, by a coordinate change, we obtain the Klein-Gordon equation in its ordinary form. However, we are determining explicit results of this background on the scalar field, since we are interested in a possible detection.

In our first analysis, we considered the presence of a hard-wall potential, where we have shown that there is the influence of the effects of the LSV on the relativistic energy levels. Then, by modifying the mass term, we inserted the Coulomb-type central potential into the Klein-Gordon equation, where we determined the energy levels of the analyzed systems, in which in turn in all cases we can note the influence of the LSV on the levels of the relativistic energy levels. In the case of the linear central potential, we have calculated the values allowed for the lower energy states of the system and shown that there is also influence of the LSV. Then, we extend our analysis considering the presence of the Coulom-type potential plus the linear potential and show that relativistic energy allowed for lower energy state is affected by the effects of the LSV. In addition, the influence of the linear central potential and of the Coulomb-type plus linear central potential on the scalar field restricts the values of the parameter related to the linear central potential to a set of values that are established by the quantum numbers of the system which allow us to obtain a polynomial solution to the biconfluent Heun series. We also can note that because the symmetry is cylindrical, the scalar field is subject to the effects of the axial central potentials only in the $x y$-plane.

It is worth mentioning that the background vector field introduced in the Klein-Gordon equation can be considered more general, that is, where all its components are nonzero. It is in our interest as future perspectives to analyze this more general case on the scalar field, not only for the central potentials considered in the present work, but for other interactions and external effects, for example, the Klein-Gordon oscillator [100-104], the Landau quantization [105], the Aharonov-Bohm effect for bound states [106], and thermodynamic properties [99, 107-111].

\section{Data Availability}

There is no use of data in this manuscript.

\section{Conflicts of Interest}

The authors declare that they have no conflicts of interest.

\section{Acknowledgments}

The authors would like to thank the Brazilian agency CNPq for financial support. R. L. L. Vitória was supported by the CNPq Project no. 150538/2018-9.

\section{References}

[1] R. Pohl, F. Nez, L. M. Fernandes et al., "Laser spectroscopy of muonic deuterium," Science, vol. 353, pp. 669-673, 2016.

[2] A. Songaila and L. L. Cowie, "Fine-structure variable?" Nature, vol. 398, no. 6729, pp. 667-668, 1999.

[3] L. L. Cowie and A. Songaila, “The inconstant constant?" Nature, vol. 428, no. 6979, pp. 132-133, 2004.

[4] V. A. Kostelecký and S. Samuel, "Spontaneous breaking of Lorentz symmetry in string theory," Physical Review D: Particles, Fields, Gravitation and Cosmology, vol. 39, p. 683, 1989.

[5] D. Colladay and V. A. Kostelecký, "CPT violation and the standard model," Physical Review D: Particles, Fields, Gravitation and Cosmology, vol. 55, article 6760, 1997.

[6] D. Colladay and V. A. Kostelecký, "Lorentz-violating extension of the standard model," Physical Review D: Particles, Fields, Gravitation and Cosmology, vol. 58, Article ID 116002, 1998.

[7] D. Colladay and V. Kostelecký, "Cross sections and lorentz violation,” Physics Letters B, vol. 511, no. 2-4, pp. 209-217, 2001.

[8] R. Lehnert, "Threshold analyses and Lorentz violation," Physical Review D, vol. 68, Article ID 085003, 2003.

[9] R. Lehnert, "Dirac theory within the standard-model extension," Journal of Mathematical Physics, vol. 45, no. 8, pp. 33993412, 2004.

[10] B. Altschul, "Compton scattering in the presence of Lorentz and CPT violation,” PHysical Review D, vol. 70, Article ID 056005, 2004.

[11] G. M. Shore, "Strong equivalence, Lorentz and CPT violation, anti-hydrogen spectroscopy and gamma-ray burst polarimetry," Nuclear Physics B, vol. B717, pp. 86-118, 2005.

[12] S. Aghababaei and M. Haghighat, "Muon anomalous magnetic moment in the standard model extension," Physical Review D, vol. 96, Article ID 115028, 2017.

[13] R. Bluhm, V. A. Kostelecký, and C. D. Lane, "CPT and Lorentz Tests with Muons," Physical Review Letters, vol. 84, no. 6, pp. 1098-1101, 2000.

[14] R. Bluhm, V. A. Kostelecký, C. D. Lane, and N. Russell, "Clockcomparison tests of Lorentz and CPT symmetry in space," Physical Review Letters, vol. 88, no. 9, Article ID 090801, 2002. 
[15] S. M. Carrol, G. B. Field, and R. Jackiw, "Limits on a Lorentzand parity-violating modification of electrodynamics," Physical Review D: Particles, Fields, Gravitation and Cosmology, vol. 41, no. 4, pp. 1231-1240, 1990.

[16] A. A. Andrianov, D. Espriu, P. Giacconi, and R. Soldati, "Anomalous positron excess from Lorentz-violating QED," Journal of High Energy Physics, vol. 2009, no. 09, article 057, 2009.

[17] J. Alfaro, A. A. Andrianov, M. Cambiaso, P. Giacconi, and R. Soldati, "Bare and induced lorentz and cpt invariance violations in QED," International Journal of Modern Physics A, vol. 25, no. 16, pp. 3271-3306, 2010.

[18] Y. Gomes and P. Malta, "Laboratory-based limits on the Carroll-Field-Jackiw Lorentz-violating electrodynamics," Physical Review D: Particles, Fields, Gravitation and Cosmology, vol. 94, no. 2, Article ID 025031, 2016.

[19] A. Martn-Ruiz and C. A. Escobar, "Local effects of the quantum vacuum in Lorentz-violating electrodynamics," Physical Review D: Particles, Fields, Gravitation and Cosmology, vol. 95, no. 3, Article ID 036011, 2017.

[20] R. Lehnert and R. Potting, "Vacuum čerenkov radiation," Physical Review Letters, vol. 93, no. 11, Article ID 110402, 2004.

[21] R. Lehnert and R. Potting, "Publisher's note: čerenkov effect in Lorentz-violating vacua," Physical Review D: Particles, Fields, Gravitation and Cosmology, vol. 70, no. 12, Article ID 125010, 2004.

[22] C. Kaufhold and F. R. Klinkhamer, "Vacuum Cherenkov radiation and photon triple-splitting in a Lorentz-noninvariant extension of quantum electrodynamics," Nuclear Physics B, vol. 734, no. 1-2, pp. 1-23, 2006.

[23] B. Altschul, "Cerenkov radiation in a Lorentz-violating and birefringent vacuum," Physical Review D, vol. 75, no. 10, Article ID 105003, 2007.

[24] B. Altschul, "Vacuum Čerenkov radiation in lorentz-violating theories without CPT Violation," Physical Review Lett, vol. 98, Article ID 041603, 2007.

[25] C. Kaufhold and F. R. Klinkhamer, "Vacuum Cherenkov radiation in spacelike Maxwell-Chern-Simons theory," Physical Review D: Particles, Fields, Gravitation and Cosmology, vol. 76, no. 2, Article ID 025024, 2007.

[26] B. Altschul, "Finite duration and energy effects in Lorentzviolating vacuum Cerenkov radiation," Nuclear Physics B, vol. 796, no. 1-2, pp. 262-273, 2008.

[27] C. A. Escobar and M. A. G. Garcia, "Full CPT-even photon sector of the standard model extension at finite temperature," Physical Review D, vol. 92, Article ID 025034, 2015.

[28] A. Martn-Ruiz and C. A. Escobar, "Casimir effect between ponderable media as modeled by the standard model extension," Physical Review D, vol. 94, no. 7, Article ID 076010, 2016.

[29] F. R. Klinkhamer and M. Risse, "Ultrahigh-energy cosmic-ray bounds on nonbirefringent modified Maxwell theory," Physical Review D: Particles, Fields, Gravitation and Cosmology, vol. 77, no. 1, Article ID 016002, 2008.

[30] F. R. Klinkhamer and M. Risse, "Addendum: Ultrahigh-energy cosmic-ray bounds on nonbirefringent modified Maxwell theory," Physical Review D: Particles, Fields, Gravitation and Cosmology, vol. 77, no. 11, Article ID 117901, 2008.

[31] F. R. Klinkhamer and M. Schreck, "New two-sided bound on the isotropic Lorentz-violating parameter of modified Maxwell theory," Physical Review D: Particles, Fields, Gravitation and Cosmology, vol. 78, no. 8, Article ID 085026, 2008.
[32] A. Moyotl, H. Novales-Sánchez, J. J. Toscano, and E. S. Tututi, "Gauge invariant electromagnetic properties of fermions induced by CPT-violation in the standard model extension," International Journal of Modern Physics A, vol. 29, no. 8, Article ID 1450039, 2014.

[33] M. Schreck, "Analysis of the consistency of parity-odd nonbirefringent modified Maxwell theory," Physical Review D, vol. 86, no. 6, Article ID 065038, 2012.

[34] B. Agostini, F. Barone, F. Barone, P. Gaete, and J. Helayël-Neto, "Consequences of vacuum polarization on electromagnetic waves in a Lorentz-symmetry breaking scenario," Physics Letters B, vol. 708, no. 1-2, pp. 212-215, 2012.

[35] L. C. Brito, H. G. Fargnoli, and A. P. Baêta Scarpelli, "Aspects of quantum corrections in a Lorentz-violating extension of the Abelian Higgs model," Physical Review D: Particles, Fields, Gravitation and Cosmology, vol. 87, no. 12, Article ID 125023, 2013.

[36] T. Mariz, J. R. Nascimento, E. Passos, R. F. Ribeiro, F. A. Brito, and J. High Energy, "A remark on Lorentz violation at finite temperature," Journal of High Energy Physics, vol. 2005, no. 10, article 019, 2005.

[37] J. R. Nascimento, E. Passos, A. Yu. Petrov, and F. A. Brito, "Lorentz-CPT violation, radiative corrections and finite temperature," Journal of High Energy Physics, vol. 2007, no. 6, article 016, 2007.

[38] A. P. Scarpelli, M. Sampaio, M. C. Nemes, and B. Hiller, "Gauge invariance and the CPT and Lorentz violating induced Chern-Simons-like term in extended QED," The European Physical Journal C, vol. 56, no. 4, pp. 571-578, 2008.

[39] F. A. Brito, J. R. Nascimento, E. Passos, and A. Y. Petrov, "The ambiguity-free four-dimensional Lorentz-breaking ChernSimons action," Physics Letters. B. Particle Physics, Nuclear Physics and Cosmology, vol. 664, no. 1-2, pp. 112-115, 2008.

[40] F. A. Brito, L. S. Grigorio, M. S. Guimaraes, E. Passos, and C. Wotzasek, "Induced Chern-Simons-like action in Lorentzviolating massless QED," Physical Review D: Particles, Fields, Gravitation and Cosmology, vol. 78, no. 12, Article ID 125023, 2008.

[41] O. M. Del Cima, J. M. Fonseca, D. H. Franco, and O. Piguet, "Lorentz and CPT violation in QED revisited: a missing analysis," Physics Letters B, vol. 688, no. 2-3, pp. 258-262, 2010.

[42] V. A. Kostelecký and M. Mewes, "Electrodynamics with Lorentz-violating operators of arbitrary dimension," Physical Review D, vol. 80, no. 1, Article ID 015020, 2009.

[43] M. Mewes, "Optical-cavity tests of higher-order Lorentz violation," Physical Review D, vol. 85, no. 11, Article ID 116012, 2012.

[44] M. Schreck, "Quantum field theoretic properties of Lorentzviolating operators of nonrenormalizable dimension in the photon sector," Physical Review D: Particles, Fields, Gravitation and Cosmology, vol. 89, no. 10, Article ID 105019, 17 pages, 2014.

[45] V. A. Kostelecký and M. Mewes, "Fermions with Lorentzviolating operators of arbitrary dimension," Physical Review D: Particles, Fields, Gravitation and Cosmology, vol. 88, no. 9, Article ID 096006, 2013.

[46] M. Schreck, "Quantum field theoretic properties of Lorentzviolating operators of nonrenormalizable dimension in the fermion sector," Physical Review D, vol. 90, no. 8, Article ID 085025, 2014.

[47] R. C. Myers and M. Pospelov, "Ultraviolet modifications of dispersion relations in effective field theory," Physical Review Letters, vol. 90, no. 21, Article ID 211601, 4 pages, 2003. 
[48] C. M. Reyes, L. F. Urrutia, and J. D. Vergara, "Quantization of the Myers-Pospelov model: The photon sector interacting with standard fermions as a perturbation of QED," Physical Review D: Particles, Fields, Gravitation and Cosmology, vol. 78, no. 12, Article ID 125011, 2008.

[49] J. Lopez-Sarrion and C. M. Reyes, "Microcausality and quantization of the fermionic Myers-Pospelov model," The European Physical Journal C, vol. 72, no. 9, article 2150, 2012.

[50] C. Reyes, L. Urrutia, and J. Vergara, "The photon sector in the quantum Myers-Pospelov model: An improved description," Physics Letters B, vol. 675, no. 3-4, pp. 336-339, 2009.

[51] C. M. Reyes, "Causality and stability for Lorentz-CPT violating electrodynamics with dimension-5 operators," Physical Review $D$, vol. 82, Article ID 125036, 26 pages, 2010.

[52] C. M. Reyes, "Unitarity in higher-order Lorentz-invariance violating QED," Physical Review D, vol. 87, no. 12, Article ID 125028, 7 pages, 2013.

[53] K. Bakke and H. Belich, "Quantum holonomies based on the Lorentz-violating tensor background," Journal of Physics G: Nuclear and Particle Physics, vol. 40, no. 6, Article ID 065002, 2013.

[54] H. Belich, T. Costa-Soares, M. A. Santos, and M. T. D. Orlando, "Lorentz symmetry violation," Revista Brasileira de Ensino de Física, vol. 29, no. 1, pp. 57-64, 2007.

[55] K. Bakke and H. Belich, "Relativistic Landau-He-McKellarWilkens quantization and relativistic bound states solutions for a Coulomb-like potential induced by the Lorentz symmetry breaking effects," Annals of Physics, vol. 333, pp. 272-281, 2013.

[56] R. Casana, M. M. Ferreira, R. V. Maluf, and F. E. P. dos Santos, "Radiative generation of the CPT-even gauge term of the SME from a dimension-five nonminimal coupling term," Physics Letters, vol. 726, pp. 815-819, 2013.

[57] R. Casana, M. M. Ferreira, E. da Hora, and A. B. F. Neves, "Maxwell-Chern-Simons vortices in a CPT-odd Lorentzviolating Higgs electrodynamics," The European Physical Journal C, vol. 74, article 3064, 2014.

[58] R. Casana, M. M. Ferreira, and P. F. E. dos Santos, "GuptaBleuler quantization of the anisotropic parity-even and C P Teven electrodynamics of a standard model extension," Physical Review D, vol. 90, Article ID 105025, 2014.

[59] R. Casana, C. F. Farias, and M. M. Ferreira, "Topological selfdual configurations in a Lorentz-violating gauged $O(3)$ sigma model," Physical Review D: Particles, Fields, Gravitation and Cosmology, vol. 92, Article ID 125024, 2015.

[60] R. Casana, M. M. Ferreira, V. E. Mouchrek-Santos, and E. O. Silva, "Generation of geometrical phases and persistent spin currents in 1-dimensional rings by Lorentz-violating terms," Physics Letters B, vol. 746, pp. 171-177, 2015.

[61] G. Gazzola, H. G. Fargnoli, A. P. Baêta Scarpelli et al., "QED with minimal and nonminimal couplings: on the quantum generation of Lorentz-violating terms in the pure photon sector," Journal of Physics G: Nuclear and Particle Physics, vol. 39, Article ID 035002, 2012.

[62] K. Bakke and H. Belich, "A Landau-type quantization from a Lorentz symmetry violation background with crossed electric and magnetic fields," Journal of Physics G: Nuclear and Particle Physics, vol. 42, Article ID 095001, 2015.

[63] K. Bakke and H. Belich, Spontaneous Lorentz Symmetry Violation and Low Energy Scenarios, LAMBERT Academic Publishing, Saarbrücken, Germany, 2015.
[64] K. Bakke and H. Belich, "On a relativistic scalar particle subject to a Coulomb-type potential given by Lorentz symmetry breaking effects," Annals of Physics, vol. 360, pp. 596-604, 2015.

[65] K. Bakke and H. Belich, "On the harmonic-type and linear-type confinement of a relativistic scalar particle yielded by Lorentz symmetry breaking effects," Annals of Physics, vol. 373, pp. 115122, 2016.

[66] R. L. L. Vitória, H. Belich, and K. Bakke, "A relativistic quantum oscillator subject to a Coulomb-type potential induced by effects of the violation of the Lorentz symmetry," The European Physical Journal Plus, vol. 132, article 25, 2017.

[67] R. L. L. Vitória, H. Belich, and K. Bakke, "Coulomb-type interaction under lorentz symmetry breaking effects," Advances in High Energy Physics, vol. 2017, Article ID 6893084, 5 pages, 2017.

[68] M. Gomes, J. R. Nascimento, A. Petrov et al., "On the aetherlike Lorentz-breaking actions," Physical Review, vol. 81, Article ID 045018, 16 pages, 2010.

[69] M. B. Cruz, E. R. de Mello, and A. Y. Petrov, "Casimir effects in Lorentz-violating scalar field theory," Physical Review D: Particles, Fields, Gravitation and Cosmology, vol. 96, no. 4, 045019, 12 pages, 2017.

[70] G. Arfken and H. J. Weber, Mathematical Methods for Physicists, Elsevier Academic Press, New York, NY, USA, 6th edition, 2005.

[71] K. Bakke, "On the rotating effects and the Landau-AharonovCasher system subject to a hard-wall confining potential in the cosmic string spacetime," International Journal of Theoretical Physics, vol. 54, no. 7, pp. 2119-2126, 2015.

[72] K. Bakke, "A geometric approach to confining a Dirac neutral particle in analogous way to a quantum dot," The European Physical Journal B, vol. 85, article 354, 2012.

[73] L. C. N. Santos and C. C. Barros, "Relativistic quantum motion of spin-0 particles under the influence of noninertial effects in the cosmic string spacetime," The European Physical Journal C, vol. 78, article 13, 2018.

[74] L. B. Castro, "Noninertial effects on the quantum dynamics of scalar bosons," The European Physical Journal C, vol. 76, article 61, 2016.

[75] R. L. Vitória and K. Bakke, "Aharonov-Bohm effect for bound states in relativistic scalar particle systems in a spacetime with a spacelike dislocation," International Journal of Modern Physics D: Gravitation, Astrophysics, Cosmology, vol. 27, no. 2, Article ID 1850005, 2018.

[76] R. L. L. Vitória and K. Bakke, "Rotating effects on the scalar field in the cosmic string spacetime, in the spacetime with spacelike dislocation and in the spacetime with a spiral dislocation," European Physical Journal C, vol. 78, no. 175, 2018.

[77] W. Greiner, Relativistic Quantum Mechanics: Wave Equations, Springer, Berlin, Germany, 3rd edition, 2000.

[78] M. K. Bahar and F. Yasuk, "Exact solutions of the massdependent klein-gordon equation with the vector quarkantiquark interaction and harmonic oscillator potential," Advances in High Energy Physics, vol. 2013, Article ID 814985, 6 pages, 2013.

[79] E. R. Figueiredo Medeiros and E. R. Bezerra de Mello, "Relativistic quantum dynamics of a charged particle in cosmic string spacetime in the presence of magnetic field and scalar potential," The European Physical Journal C, vol. 72, article 2051, 2012.

[80] R. L. L. Vitória, C. Furtado, and K. Bakke, "Linear confinement of a scalar particle in a Gödel-type spacetime," The European Physical Journal C, vol. 78, no. 44, 2018. 
[81] A. L. Cavalcanti de Oliveira and E. R. Bezerra de Mello, "Exact solutions of the Klein-Gordon equation in the presence of a dyon, magnetic flux and scalar potential in the spacetime of gravitational defects," Classical and Quantum Gravity, vol. 23, no. 17, pp. 5249-5263, 2006.

[82] K. Bakke and C. Furtado, "On the Klein-Gordon oscillator subject to a Coulomb-type potential," Annals of Physics, vol. 355, pp. 48-54, 2015.

[83] R. L. L. Vitória, C. Furtado, and K. Bakke, "On a relativistic particle and a relativistic position-dependent mass particle subject to the klein-gordon oscillator and the coulomb potential," Annals of Physics, vol. 370, no. 128, pp. 128-136, 2016.

[84] G. Soff, B. Müller, J. Rafelski, and W. Greiner, "Solution of the dirac equation for scalar potentials and its implications in atomic physics," Zeitschrift für Naturforschung A, vol. 28, no. 9, pp. 1389-1396, 1973.

[85] H. Asada and T. Futamase, "Propagation of gravitational waves from slow motion sources in a Coulomb-type potential," Physical Review D: Particles, Fields, Gravitation and Cosmology, vol. 56, no. 10, pp. R6062-R6066, 1997.

[86] P. Gribi and E. Sigmund, "Exact solutions for a quasi-onedimensional Coulomb-type potential," Physical Review B: Condensed Matter and Materials Physics, vol. 44, no. 8, pp. 35373549, 1991.

[87] F. Gesztesy and B. Thaller, "Born expansions for Coulomb-type interactions," Journal of Physics A: Mathematical and General, vol. 14, no. 3, pp. 639-657, 1981.

[88] J. A. Reyes and M. del Castillo-Mussot, "1D Schrödinger equations with Coulomb-type potentials," Journal of Physics A: Mathematical and General, vol. 32, no. 10, pp. 2017-2025, 1999.

[89] A. De Souza Dutra, "Conditionally exactly soluble class of quantum potentials," Physical Review A: Atomic, Molecular and Optical Physics, vol. 47, no. 4, pp. R2435-R2437, 1993.

[90] S. M. Ikhdair and M. Hamzavi, "Effects of external fields on a two-dimensional Klein-Gordon particle under pseudoharmonic oscillator interaction," Chinese Physics B, vol. 21, p. 110302, 2012.

[91] S. M. Ikhdair, B. J. Falaye, and M. Hamzavi, "Nonrelativistic molecular models under external magnetic and AB flux fields," Annals of Physics, vol. 353, pp. 282-298, 2015.

[92] I. I. Guseinov and B. A. Mamedov, "Evaluation of multicenter one-electron integrals of noninteger u screened Coulomb type potentials and their derivatives over noninteger $\mathrm{n}$ Slater orbitals," The Journal of Chemical Physics, vol. 121, no. 4, article 1649, 2004.

[93] I. I. Guseinov, "Unified treatment of multicenter integrals of integer and noninteger u Yukawa-type screened Coulomb type potentials and their derivatives over Slater orbitals," The Journal of Chemical Physics, vol. 120, no. 20, pp. 9454-9457, 2004.

[94] R. L. L. Vitória and K. Bakke, "Relativistic quantum effects of confining potentials on the Klein-Gordon oscillator," The European Physical Journal Plus, vol. 131, no. 36, 2016.

[95] R. L. L. Vitória and K. Bakke, "Torsion effects on a relativistic position-dependent mass system," General Relativity and Gravitation, vol. 48, article 161, 2016.

[96] R. F. Ribeiro and K. Bakke, "On the Majorana fermion subject to a linear confinement," Annals of Physics, vol. 385, pp. 36-39, 2017.

[97] A. Ronveaux, Heun's Differential Equations, Oxford University Press, Oxford, UK, 1995.
[98] C. Furtado, "Landau levels in the presence of disclinations," Physics Letters A, vol. 195, no. 1, pp. 90-94, 1994.

[99] M. Eshghi and H. Mehraban, "Study of a 2D charged particle confined by a magnetic and $\mathrm{AB}$ flux fields under the radial scalar power potential," The European Physical Journal Plus, vol. 132, article 121, 2017.

[100] S. Bruce and P. Minning, "The klein-gordon oscillator," Il Nuovo Cimento A, vol. 106, no. 5, pp. 711-713, 1993.

[101] V. V. Dvoeglazov, "The Klein-Gordon oscillator," Il Nuovo Cimento A, vol. 107, pp. 1413-1418, 1994

[102] N. A. Rao and B. A. Kagali, "Energy profile of the onedimensional Klein-Gordon oscillator," Physica Scripta, vol. 77, Article ID 015003, 2008.

[103] A. Boumali, A. Hafdallah, and A. Toumi, "Comment on 'Energy profile of the one-dimensional Klein-Gordon oscillator," Physica Scripta, vol. 84, Article ID 037001, 2011.

[104] B. Mirza and M. Mohadesi, "The Klein-Gordon and the Dirac oscillators in a noncommutative space," Communications in Theoretical Physics, vol. 42, no. 5, pp. 664-668, 2004.

[105] L. D. Landau and E. M. Lifshitz, Quantum Mechanics: The Nonrelativistic Theory, Pergamon, Oxford, UK, 3rd edition, 1977.

[106] Y. Aharonov and D. Bohm, "Significance of electromagnetic potentials in the quantum theory," Physical Review A: Atomic, Molecular and Optical Physics, vol. 115, no. 3, article 485, 1959.

[107] X.-Q. Song, C.-W. Wang, and C.-S. Jia, "Thermodynamic properties for the sodium dimer," Chemical Physics Letters, vol. 673, pp. 50-55, 2017.

[108] H. Hassanabadi and M. Hosseinpour, "Thermodynamic properties of neutral particle in the presence of topological defects in magnetic cosmic string background," The European Physical Journal C, vol. 76, article 553, 2016.

[109] A. N. Ikot, B. C. Lutfuoglu, M. I. Ngwueke, M. E. Udoh, S. Zare, and H. Hassanabadi, "Klein-Gordon equation particles in exponential-type molecule potentials and their thermodynamic properties in D dimensions," The European Physical Journal Plus, vol. 131, article 419, 2016.

[110] B. Hamil and M. Merad, "Dirac and Klein-Gordon oscillators on anti-de Sitter space," The European Physical Journal Plus, vol. 133, pp. 133-174, 2018.

[111] B. Khosropour, "Statistical aspects of the Klein-Gordon oscillator in the frame work of GUP," Indian Journal of Physics, vol. 92 , no. 1, pp. 43-47, 2018. 

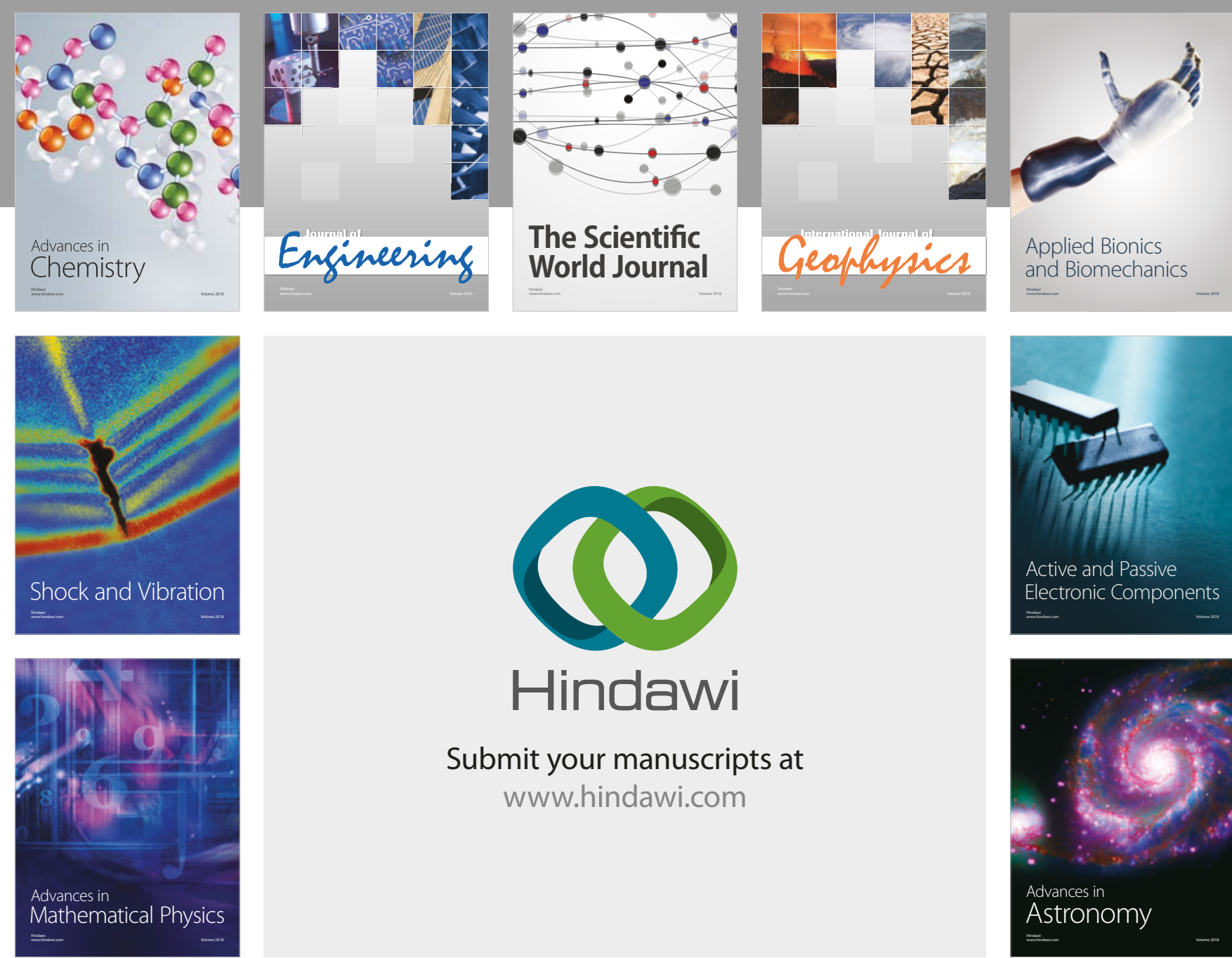

Submit your manuscripts at

www.hindawi.com

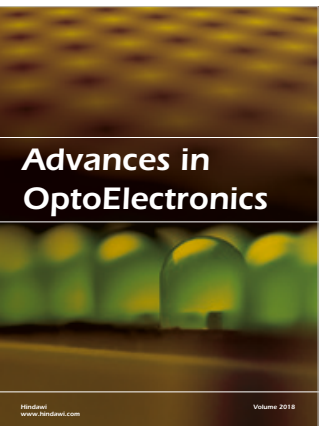

\section{Rotcting Machinery}
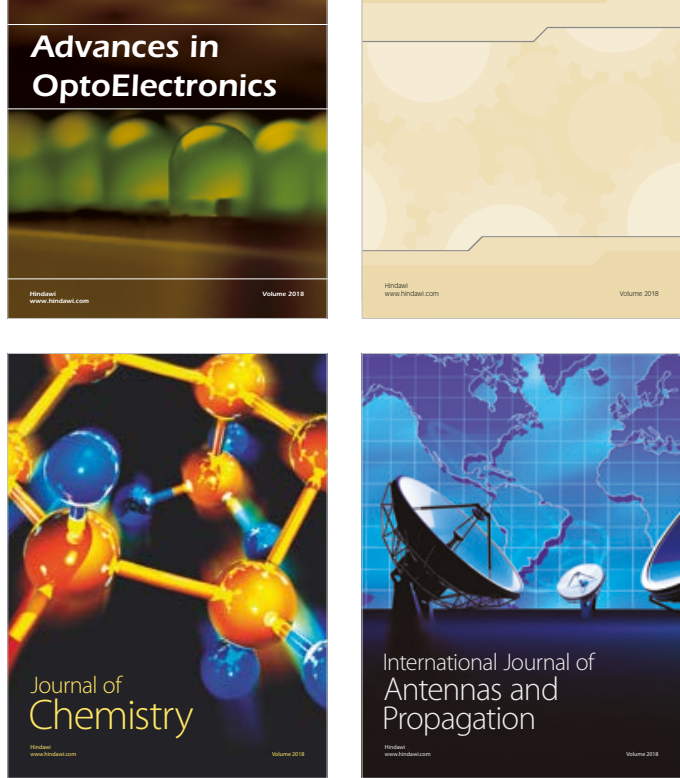

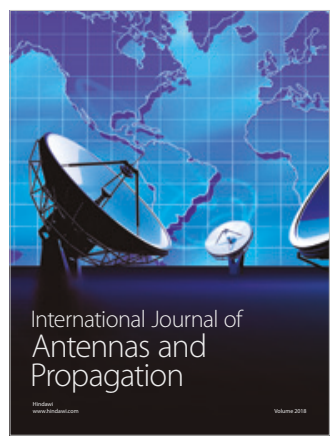

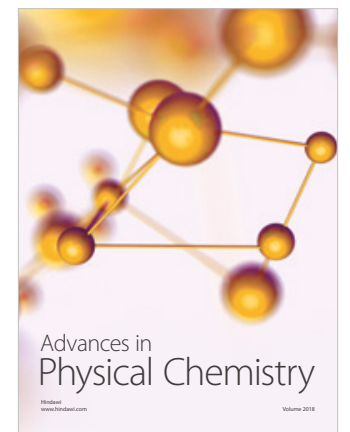

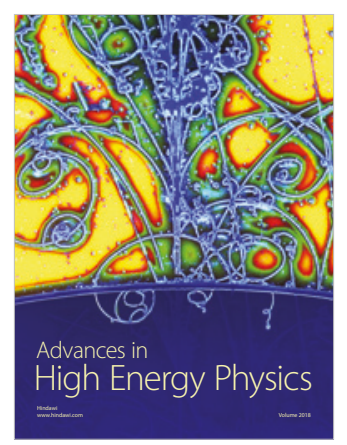

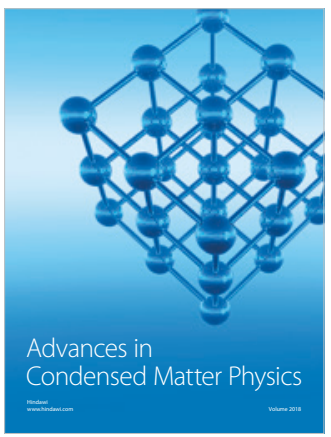

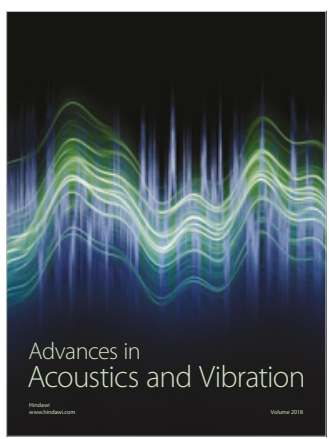

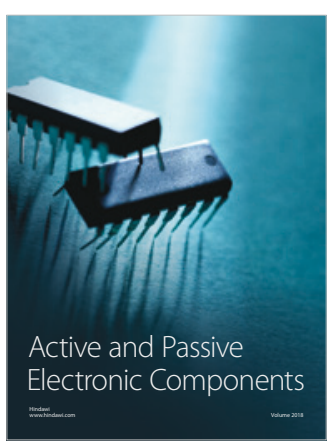
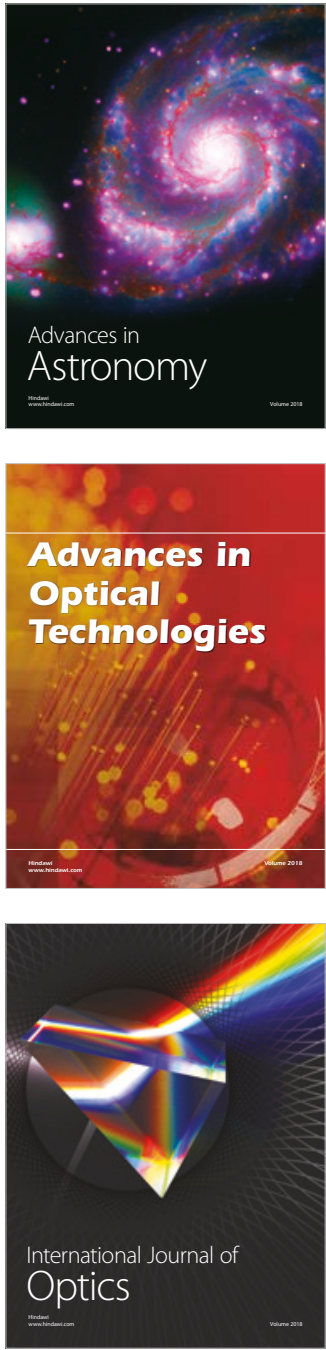